\title{
El Plexo Coroideo: Historia, Estructura, Función, Etimología y Propuesta de Cambio Terminológico
}

\author{
The Choroid Plexus: History, Structure, Function, Etymology \\ and Proposal for Terminological Change
}

Jorge Eduardo Duque Parra ${ }^{1,2}$; Steven Castillo Ramírez ${ }^{1}$ \& José Fernando Marín Arias ${ }^{3}$

DUQUE, P. J. E.; CASTILLO, R. S. \& MARÍN, A. J. F. El plexo coroideo: historia, estructura, función, etimología y propuesta de cambio terminológico. Int. J. Morphol., 38(6):1577-1579, 2020.

RESUMEN: El plexo coroideo es una estructura del sistema nervioso central vinculada con la producción de parte del líquido cerebroespinal. Su conocimiento deriva de los tiempos de Herophilus de Calcedonia a quien se atribuye su nombre, el cual ha perdurado, asumiendo el componente coroideo como la forma de la piel de los animales después del proceso de curtido y preparado para diferentes usos en la industria. Se propone utilizar como nombre, uno estructural: el de plexo ventricular ependimario, para reemplazar el tradicional coroideo que indica similitud al cuero o piel encurtida de los animales.

PALABRAS CLAVE: Plexo coroideo; Neuroanatomía; Terminologia Anatomica.

\section{INTRODUCCIÓN}

El descubrimiento del plexo coroideo en los ventrículos encefálicos fue atribuido a Herophilus de Calcedonia (335-280 a.n.e) por Galeno de Pergamo (130-210), que lo denominó "choroid menix". Rufo de Éfeso (siglo I) sugirió para esa estructura el término "túnica coroidea" para describir el epéndimo como plexo coroideo (Dohrmann, 1970), aunque el concepto contemporáneo reconoce en las células ependimarias solo uno de sus componentes (GhersiEgea et al., 2018). Por largo tiempo no se hicieron referencias al respecto de estos elementos del sistema nervioso, hasta que Andreas Vesalius -1514-1564- describe macroscópicamente estos plexos en los ventrículos laterales (Vesalius, 1555), posteriormente Thomas Willis -16211675- refiere el plexo coroideo del ventrículo IV (Willis, 1664) y H Ridley lo hace para el III ventrículo (Ridley, 1695).

El plexo coroideo es una estructura de tejido neuro conectivo vascular localizada en el sistema ventricular encefálico (Fig. 1), ubicado en los ventrículos laterales, tercero y cuarto. Se constituye por epitelio cuboideo simple con numerosos cilios (del Bigio, 2010), estas células cuboideas se continúan con el revestimiento ependimario envolviéndose en torno al estroma conectivo, cuyos vasos sanguíneos sin capas musculares en sus paredes, lo pene- tran formando asas capilares fenestradas juxtapuestas al delgado epitelio (Ghersi-Egea et al.). Además, el plexo posee fibras nerviosas mielinicas y amielinicas (Redzic \& Segal, 2004). El epitelio conforma la barrera sangre-fluido cerebroespinal (Emerich et al., 2004; Ghersi-Egea et al.), tiene presencia de algunas microvellosidades finas e irregulares que aumentan la superficie de la célula, factor importante para su actividad secretora (Tamega et al., 2000) como la principal fuente de producción de líquido cerebro espinal (Lun et al., 2015), aunque algunos autores creen que la producción extra coroidea de líquido cerebroespinal depende sobre todo del epéndimo y del parénquima encefálico (Milhorat et al., 1971). Esta producción de líquido cerebroespinal sirve para el mantenimiento del medio extracelular en parte, al secretar varios factores de crecimiento (Emerich et al.) con su papel activo en la regulación de las células vástago neuronales (Lun et al.), además de inspeccionar inmunológicamente el encéfalo, secretando polipéptidos y participando en su reparación durante los traumas (Thanos et al., 2010). El plexo coroideo clínicamente se relaciona con diversas alteraciones vasculares como hemorragias intra ventriculares, subaracnoideas e intra cerebrales (Xiang et al., 2017) además de tumores (Miller et al, 2019).

\footnotetext{
${ }^{1}$ Universidad de Caldas. Programa de Medicina. Universidad de Caldas. Manizales, Colombia.

${ }^{2}$ Departamento de Ciencias Básicas. Programa de Medicina. Universidad de Manizales, Colombia.

${ }^{3}$ Departamento de Salud Pública. Universidad de Caldas. Manizales, Colombia.
} 


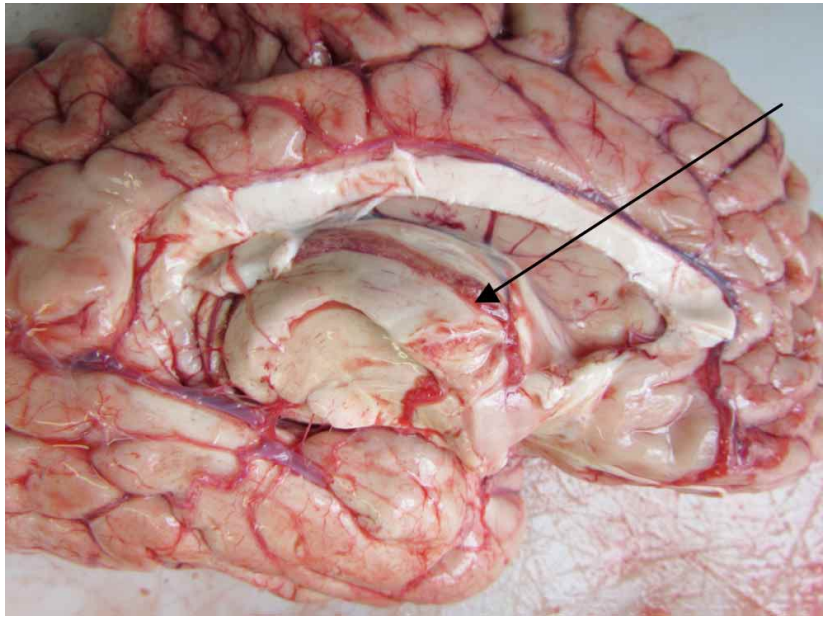

Fig. 1. Corte sagital de un encéfalo humano de adulto en el que se distingue con la punta de la flecha de línea negra el plexo coroideo del tercer ventrículo sobre el tálamo. como bolsas donde metemos el pie, similar a alforjas de cuero (Franco Rodríguez, 2013); también se relaciona con coriarius: el término dado al curtidor del cuero y con coriáceo: lo que es de cuero (Salvá, 1843).

El término que define estas estructuras en los ventrículos encefálicos como plexo coroideo, deriva de Plexus choroideus indicado en la Terminologia Anatomica internacional en el capítulo sobre el sistema nervioso, numeral 5409 (FIPAT, 2019) y en Terminologia Neuroanatomica (FIPAT, 2017) numeral 326 cuyo componente Plexus asimilamos a plexo -del latín-, entendido como una red o entrelazado, para el caso anatómico de filamentos vasculares (Cadavid Restrepo, 1942) conectivos y nerviosos. El término coroideo deriva del griego corion -chorion-, en latín corium, que se corresponde a cuero (Boettner, 1942; Mascaró y Porcar, 1983) (Fig. 2), lo que equivale a la piel o pellejo de los animales después de curtido y preparado para múltiples usos en la industria (Mascaró y Porcar), término vinculado también con el saquillo de cuero en que se deposita el dinero, dicho en latín de varias maneras, entre ellas bursa y corium.
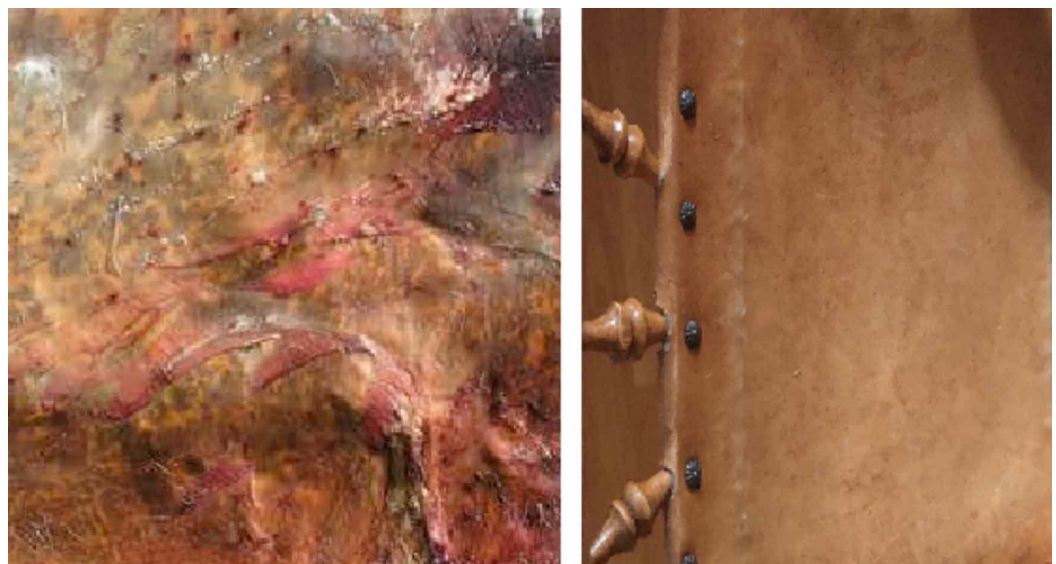

Fig. 2. Piel de animal sin encurtir y cuero adaptado para el soporte del espaldar de una silla.

\section{DISCUSIÓN}

El aprendizaje de la terminología médica puede parecer inicialmente como estudiar un idioma nuevo y extraño, sin embargo, una vez que se comprenden las reglas básicas sobre cómo se forman los términos médicos mediante el desarrollo de palabras, se parecerá mucho a armar un rompecabezas. Entre las pautas generales para formar dichas palabras, se cuenta con la comprensión de las raíces de estas (Fremgen \& Frucht, 2016), pero en el término coroideo hay inconsistencia, pues, aunque a través de la historia de la morfología, se utilizó chorion -corium- desde Aristóteles 385-323 a.n.e- en referencia a la membrana o cubierta externa del embrión del pollo (Hyrtl, 1880; Aristóteles, 1992), a una cubierta del bulbo ocular (ojo) (Barnett, 1979) y a una membrana fetal (Genbacev et al., 2015), para el caso del sistema nervioso, fue desde Herophilus que se utiliza (Dohrmann) creyéndose que dicho plexo era una continuación de la piamadre, similarmente comparándolo con el corion del embrión (Hyrtl). El término coroideo hace referencia a la piel de los animales después de curtida y preparada para diferentes usos en la industria (Mascaró y Porcar). La visión de Herophilus fue macroscópica y no concuerda con que el plexo coroideo tenga apariencia de cuero (Figs. 1 y 2). Teniendo en cuenta su función y localización anatómica, el nombre plexo coroideo tampoco brinda información para poder inferirlo. Parece que después de Rufus de Efeso que lo llamó túnica coroidea (Dohrmann) sólo modificando plexo por túnica, nadie más ha analizado las consistencias e inconsistencias de este término.

Como Terminologia Anatomica propende por una comunicación más clara y precisa entre los profesionales de las ciencias médicas (Duque et al., 2016) y de otros profesionales de la salud, en el caso de los plexos coroideos, tal nombre no es preciso y manifiesta incompatibilidad con la verdadera localización en el sistema nervioso central, además no refiere su funcionamiento. La etimología de coroideo es incompatible e ilógica con la estructura, ya que en el sistema ventricular no hay cuero, por lo que se pudiera asimilar otro nombre y para el que proponemos: plexo ventricular ependimario, el cual da a indicar que es un entrelazado microscópico neuro conectivo vascular a manera de maraña - plexo- que se encuentra en los ventrículos encefálicos, y ependimario por estar asociado con células que forman este tipo de epitelio. 


\section{CONCLUSIÓN}

El plexo coroideo no tiene ningún componente similar al cuero, su denominación no hace referencia a un componente estructural del ser humano, de allí la necesidad racional de cambiar su denominación para asimilarse a una verdadera estructura en el sistema nervioso central. Se propone el término plexo ventricular ependimario, término que cumple los criterios correspondientes para su referencia morfológica y localización.

DUQUE, P. J. E.; CASTILLO, R. S. \& MARÍN, A. J. F. The choroid plexus: History, structure, function, etymology, and proposal for terminological change. Int. J. Morphol., 38(6):1577$1579,2020$.

SUMMARY: The choroid plexus is a structure of the central nervous system linked to the production of part of the cerebrospinal fluid. Its knowledge derives from the times of Herophilus of Chalcedon to whom its name is attributed, which has endured, assuming the choroidal component as the shape of the hide of animals following the tanning process preparing it for various industrial uses. It is proposed to use the structural name of the ependymal ventricular plexus to replace the traditional choroid that indicates similarity to leather tanning or cured animal hide

KEY WORDS: Choroid plexus; Neuroanatomy; Terminologia Anatomical.

\section{REFERENCIAS BIBLIOGRÁFICAS}

Aristóteles. Investigación sobre los Animales. Palli, J. (traductor). Madrid, Gredos, 1992.

Barnett, K. C. Collie eye anomaly (CEA). J. Small Anim. Pract., 20(9):53742, 1979.

Boettner, J. M. Etimología Griega y Latina para el Uso Médico. Buenos Aires, El Ateneo, 1942.

Cadavid Restrepo, T. Raíces Griegas y Latinas. Etimologías Médicas y Biológicas. Bogotá, Litografía, 1942.

Del Bigio, M. R. Ependymal cells: biology and pathology. Acta Neuropathol., 119(1):55-73, 2010.

Dohrmann, G. J. The choroid plexus: a historical review. Brain Res., 18(2):197-218, 1970.

Duque, J. E.; Barco, J. \& Aldana, J. E. La Terminología Anatómica en Colombia y el uso de epónimos en la enseñanza en medicina. Rev. Biosalud, 15(1):82-6, 2016.

Emerich, D. F.; Vasconcellos, A. V.; Elliott, R. B.; Skinner, S. J. M. \& Borlongan, C. V. The choroid plexus: function, pathology and therapeutic potential of its transplantation. Expert Opin. Biol. Ther, 4(8):1191-201, 2004.

FIPAT. Federative International Programme for Anatomical Terminology Terminologia Anatomica. 2nd ed. FIPAT.library.dal.ca. Federative International Programme for Anatomical Terminology, 2019. Disponible en: https://fipat.library.dal.ca/TA2/
FIPAT. Federative International Programme for Anatomical Terminology Terminologia Neuroanatomica.FIPAT.library.dal.ca. Federative International Programme for Anatomical Terminology, 2017.

Franco Rodríguez, J. R. Los Étimos Griegos en los Diccionarios Etimológicos de los Siglos XVI y XVII. Tesis Doctoral. Madrid, Universidad Complutense de Madrid, Facultad de Filología, 2013.

Fremgen, B. F. \& Frucht, S. S. Medical Terminology. A Living Language. Boston, Pearson, 2016.

Genbacev, O.; Vicovac, L. \& Larocque, N. The role of chorionic cytotrophoblasts in the smooth chorion fusion with parietal decidua. Placenta, 36(7):716-22, 2015.

Ghersi-Egea, J. F.; Strazielle, N.; Catala, M.; Silva-Vargas, V.; Doetsch, F. \& Engelhardt, B. Molecular anatomy and functions of the choroidal blood-cerebrospinal fluid barrier in health and disease. Acta Neuropathol., 135(3):337-61, 2018.

Hyrtl, J. Onomatologia Anatomica. Geschichte und Kritik der Anatomischen Sprache der Gegenwart. Wien, Wilhelm Braumüller, K.K. Hof- und Universitäts Buchhändler, 1880.

Lun, M. P.; Monuki, M. K. \& Lehtinen, M. K. Development and functions of the choroid plexus-cerebrospinal fluid system. Nat. Rev. Neurosci., 16(8):445-57, 2015.

Mascaró y Porcar, J. M. Diccionario Terminológico de Ciencias Médicas. $11^{\mathrm{a}}$ ed. Barcelona, Salvat, 1983.

Milhorat, T. H.; Hammock, M. K.; Fenstermacher, J. D. \& Levin, V. A. Cerebrospinal fluid production by the choroid plexus and brain. Science, 173(3994):330-2, 1971.

Miller, A. D.; Miller, C. R. \& Rossmeisl, J. H. Canine primary intracranial cancer: a clinicopathologic and comparative review of glioma, meningioma, and choroid plexus tumors. Front. Oncol., 9:1151, 2019.

Redzic, Z. B. \& Segal, M. B. The structure of the choroid plexus and the physiology of the choroid plexus epithelium. Adv. Drug. Deliv. Rev., 56(12):1695-716, 2004.

Ridley, H. The Anatomy of the Brain. London, Smith \& Walford, 1695.

Salvá, V. Diccionario Latino-Español. $5^{\mathrm{a}}$ ed. Valencia, Imprenta y Librería de Mallen y Sobrinos, 1843.

Tamega, O. J.; Tirapelli, L. F. \& Petroni, S. Scanning electron microscopy study of the choroid plexus in the monkey (Cebus apella apella). Arq. Neuropsiquiatr., 58(3B):820-5, 2000.

Thanos, C. G.; Bintz, B. \& Emerich, D. F. Microencapsulated choroid plexus epithelial cell transplants for repair of the brain. Adv. Exp. Med. Biol., 670:80-91, 2010.

Vesalius, A. De Humani corporis Fabrica. Basileae, Libri feptem, 1555.

Willis, T. Cerebri Anatome. Academia Naturalis Philofophiæ Profefforis Sidleiani. Londini, 1664.

Xiang, J.; Routhe, L. J.; Wilkinson, D. A.; Hua, Y.; Moos, T.; Xi, G. \& Keep, R. ariF. The choroid plexus as a site of damage in hemorrhagic and ischemic stroke and its role in responding to injury. Fluids Barriers CNS, 14:8, 2017.

\section{Dirección para correspondencia: \\ Jorge Eduardo Duque Parra \\ Departamento de Ciencias Básicas \\ Universidad de Caldas \\ Manizales \\ COLOMBIA}

Email: jduqueparra@yahoo.com.mx

Recibido : 15-04-2020

Aceptado: 15-06-2020 